\title{
„Wer den Zugang zur medizinischen Versorgung einschränkt, zahlt drauf“
}

Interview mit Kayvan Bozorgmehr

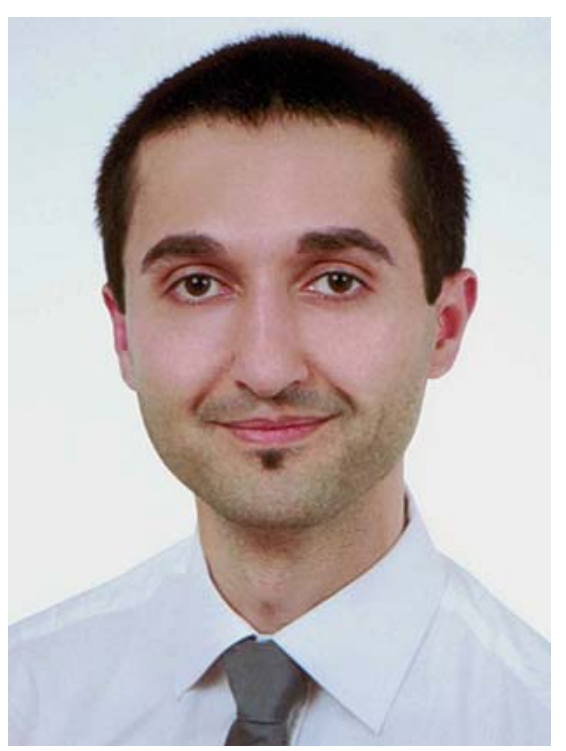

Der Epidemiologe und Public-HealthExperte Dr. med Kayvan Bozorgmehr von der Universität Heidelberg erklärt, warum die Restriktionen für den $\mathrm{Zu}$ gang zur medizinischen Versorgung nach dem hiesigen Asylbewerberleistungsgesetz nicht nur medizinisch unsinnig, sondern vermutlich auch finanziell kontraproduktiv sind.

Das Asylbewerberleistungsgesetz schränkt aktuell den Zugang zur medizinischen Versorgung für eine Reihe von Gruppen während der ersten 15 Monaten nach der Einreise ein. Sie sind Erstautor einer Studie aus dem Jahr 2015, die den Effekt dieser Einschränkungen während der ersten 15 Monate untersucht [1]. Was genau haben Sie gemacht?

Die 15 Monate sind in diesem Fall nicht ganz richtig. Unsere Studie deckt den gesamten Zeitraum von 1994 bis 2013 ab. Die Einschränkungen durch das Asylbewerberleistungsgesetz sind derzeit auf 15 Monate nach Einreise beschränkt, dies gilt aber erst seit 2015. 1993, mit der Einführung des Asylbewerberleistungsgesetzes, galt zunächst eine Frist von 12 Monaten nach Aufenthaltsbeginn, 1997 wurden es 36 Monate, 2007 änderte der Gesetzgeber das erneut, auf eine Wartezeit von 4 Jahren, 48 Monate. Seit März 2015 sind es 15 Monate. In unserem Untersuchungszeitraum galten damit immer mal wieder andere Wartezeiten.

Könnten Sie die Datengrundlage Ihrer Studie etwas erklären?

Es sind Datensätze aus der Asylbewerberleistungsstatistik. Diese Statistik wurde 1993 mit der Einführung des Asylbewerberleistungsgesetzes beschlossen. Kommunen und Landkreise haben eine Berichtspflicht, sie müssen am Ende eines jeden Jahres Informationen an die Statistischen Landesämter reichen. Es geht um viele Aspekte, darunter die Leistungsbezüge für Asylbewerber, ihre Aufenthaltssituation, Geschlecht, Alter, Herkunftsland und auch die Kosten der medizinischen Versorgung, die ja vorrangig von den Sozialämtern für die Dauer eines Asylverfahrens finanziert werden. Als aggregierte, anonymisierte Daten kommen diese Zah- 


\section{ZUR PERSON}

Dr. med Kayvan Bozorgmehr, Jahrgang 1981, leitet an der Abteilung Allgemeinmedizin und Versorgungsforschung der Universitätsklinik Heidelberg eine Arbeitsgruppe mit dem Schwerpunkt Soziale Determinanten von Gesundheit und gesundheitlicher Ungleichheit sowie Gesundheitssystemforschung.

Zusammen mit Oliver Razum von der Universität Bielefeld (siehe auch das Interview ab S. 255) hat seine Gruppe eine der bislang eher raren wissenschaftlichen Untersuchungen von Routinedaten zu den Kosten der medizinischen Versorgung von Asylbewerbern und den Einflüssen gesetzlicher Einschränkungen publiziert.

len schließlich zum Statistischen Bundesamt. Dort haben wir sie uns geholt.

\section{Ab wann genau gilt diese Statistik?}

Die Asylbewerberleistungsstatistik greift erst dann, wenn Asylbewerber von den Erstaufnahmeeinrichtungen auf Kommunen und Landkreise verteilt werden, und die Daten werden so lange erhoben, bis jemand nicht mehr Asylbewerber ist, sondern zum Beispiel eine Anerkennung des Asylstatus hat.

Mit der Ankunft in der Kommune müssen sich Asylbewerber ja auch bei dem für sie zuständigen Sozialamt einen Behandlungsschein holen, wenn sie zum Arzt wollen.

Richtig.

Woher erhalten die Sozialämter die Daten über die Kosten der Versorgung? Über die Abrechnungen mit Kassen und KVen?

Richtig, am Ende bezahlen die Sozialämter die medizinische Versorgung, rechnen dazu mit KVen und eventuell auch Kassen ab. Erst die Sozialämter werten aber derzeit überhaupt diese Abrechnungsdaten, alias Routinedaten, nach dem Merkmal Kostenträger etc. aus. KVen und Kassen haben dazu meines Wissens bislang keine eigenen Übersichten. Das ist das gleiche Problem, das wir bei den Kassen haben, wenn es um die Daten für Asylbewerber geht, die bereits länger als die Wartezeit in Deutschland sind.

Sie meinen jene Gruppe, die aktuell nach 15 Monaten Aufenthalt automatisch in die Versorgungsansprüche nach § 2 Asylbewerberleistungsgesetz wechselt und damit Anspruch auf Leistungen nach dem SGB XII hat. Diese Menschen werden jetzt direkt von Kassen betreut, genau so wie ein Hartz-IV-Empfänger bundesdeutscher Herkunft?

Richtig, Aber auch hier können bislang nur die Sozialämter die Daten herausrechnen und zum Beispiel auch Kosten auf Personenebene zuordnen.

Jetzt haben wir die Datengrundlage, was genau haben Sie untersucht?

Wir haben die Daten genau dieser beiden Gruppen verglichen - einmal jene Menschen, die während der Wartezeit der Leistungseinschränkung nach den $\S \S 4$ und 6 Asylbewerberleistungsgesetz unterliegen, und jene, die aufgrund einer Überschreiten der Befristung de facto Zugang zur Regelversorgung haben. Dabei haben wir uns vorrangig für den Vergleich der Kosten der medizinischen Versorgung interessiert.

\section{Und?}

Über den gesamten Untersuchungszeitraum, das sind ja 20 Jahre, kommen wir auf 5,57 Milliarden Euro Ausgaben für die medizinische Versorgung der Gruppe von Asylbewerbern innerhalb der Wartezeit, und 1,472 Milliarden für die 2. Gruppe.

Die Gesundheitsausgaben insgesamt lagen in Deutschland allein im Jahr 2013 bei 328 Milliarden Euro.

Ja, und verglichen damit sind die Ausgaben für die medizinische Versorgung von Asylbewerbern sehr gering - das ist eher ein Rauschen im Hintergrund.

Es wären damit zumindest bis zum Jahr 2013 insgesamt pro Jahr einige 100 Millionen Euro an Kosten für die gesundheitliche Versorgung von Asylbewerbern gewesen, mehr nicht? Haben wir damit eine Aussage darüber, wie viel die Bundesrepublik insgesamt für die gesundheitliche Versorgung von Flüchtlingen ausgibt pro Jahr?

Nein. Absolute Zahlen dazu haben wir nicht. Denn bei den medizinischen Leistun- gen in den Erstaufnahmeeinrichtungen gibt es meines Wissens keine Berichtspflicht für die Länder. Da fehlen uns Zahlen, allein schon deshalb können wir keine absolute Zahl nennen, wie viel die Medizin für Asylbewerber und Flüchtlinge insgesamt pro Jahr kostet. Zumal auch später nach Anerkennung weitere staatliche Mittel nötig sind, solange die Betroffenen noch keine sozialversicherungspflichtige Arbeit haben.

Wie viele Asylbewerber haben Sie pro Jahr in den Statistiken dabei?

Das schwankt stark ( $\triangleright$ Abb. 1) [1].

Ende 1997 waren es fast 500000 Menschen, Ende 2013 über 200000 . Jeweils bezogen auf den Stichtag 31.12. eines jeden Jahres.

\section{Woher kommt dieser Stichtag?}

Es sind die Zahlen derer, die sich an dem Tag noch im Asylverfahren befinden und die zu dem Zeitpunkt in Kommunen und Landkreisen im Asylbewerberverfahren registriert waren.

Wir haben diese Zahl allerdings immer auf das gesamte Jahr bezogen, um eine Vergleichsgrundlage zu den Kosten zu haben. Denn die Kosten der medizinischen Versorgung beziehen sich immer auf ein ganzes Jahr. Um einen Bezug auf Personen zu schaffen, sprechen wir hier von Personenjahren.

\section{Was ist das denn?}

Der Terminus ist abstrakt, ist aber ein etabliertes Verfahren in der Statistik. Personenjahre sind ein Maß in der Epidemiologie, um Bevölkerungsgrößen zu quantifizieren, wenn ich zugleich eine Zeitkomponente habe. 100 Menschen über 10 Jahre beobachtet, ist dann genauso groß wie 1000 Menschen über 1 Jahr beobachtet. Beides sind 1000 Personenjahre. Wir machen dieses Behelfskonstrukt eben unter der Annahme, dass die Personen am Jahresende jeweils alle schon für 1 Jahr im Land waren.

Das bringt einen Unsicherheitsfaktor in die Studie?

Richtig, ist aber unvermeidbar. Es ist eine Annahme, die wir treffen mussten. Und vor allem haben wir diese Annahme für beide Gruppen gestellt - was den Fehler, 


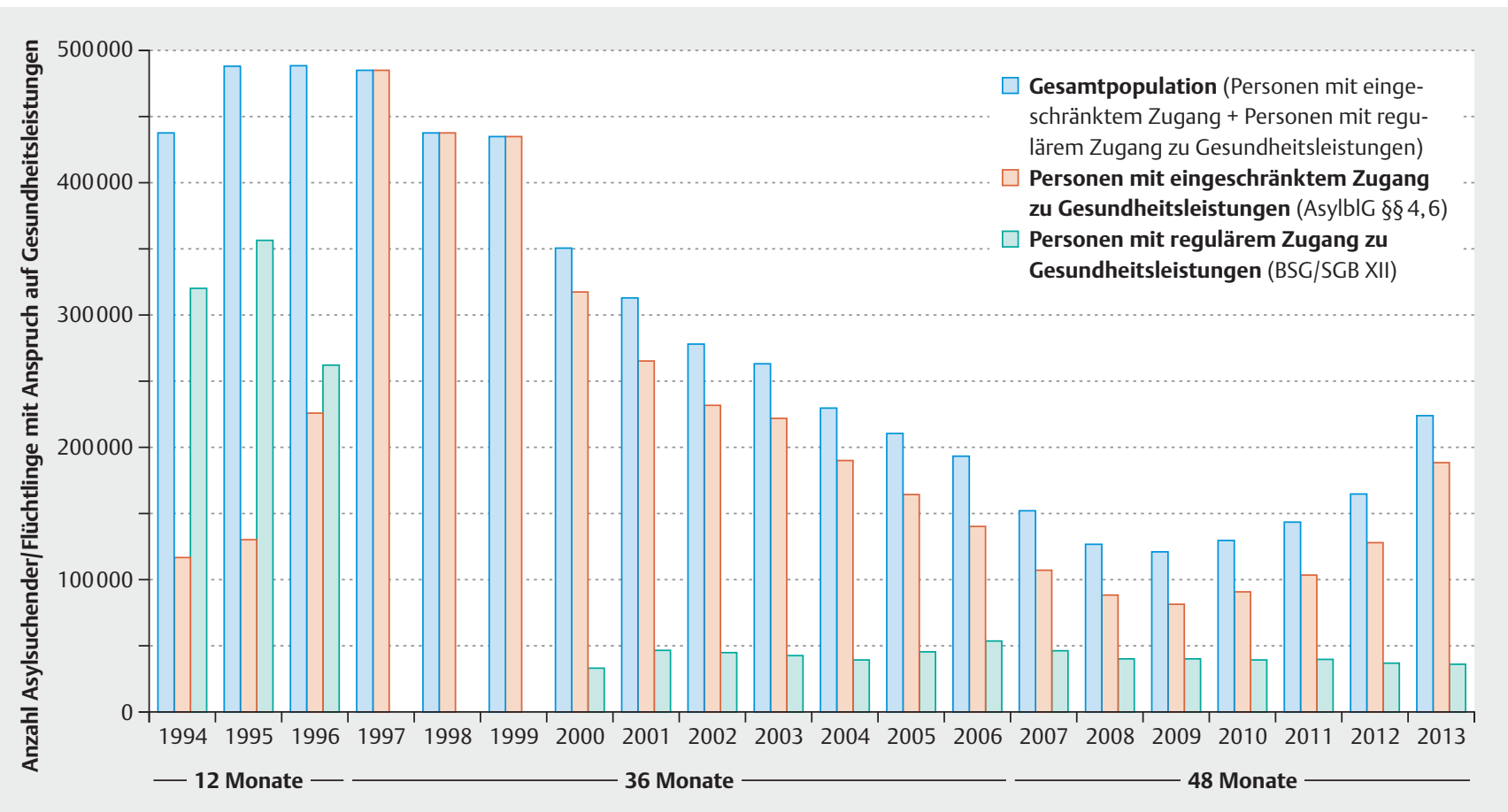

- Abb. 1 Asylsuchende und Flüchtlinge mit einem Anspruch auf Gesundheitsleistungen.

der dadurch immanent eingeschleppt wird, wieder indirekt etwas ausgleicht. Und nur über diese, ich wiederhole, statistisch legitime Annahme konnten wir auch Werte pro Kopf kalkulieren und Vergleiche anstellen.

\section{Und was ist das Ergebnis?}

Wir bewegen uns zwischen 1800 und 2000 Euro pro Jahr und Kopf - ( [1].

Damit können wir sagen, dass die Kosten der medizinischen Versorgung für Asylbewerber pro Kopf niedriger sind als der Durchschnitt der bundesdeutschen Population. Die kommt auf etwa 3000 Euro im Jahr. Das Entscheidende aber war für uns der Vergleich der beiden Gruppen von Asylbewerbern vor und nach der Einschränkung des Zugangs zur Versorgung.

\section{Was haben Sie gefunden?}

Über den Gesamtzeitraum der Untersuchung zeigt sich fast regelmäßig Jahr für Jahr, dass die Versorgung der Leute, die den eingeschränkten Zugang zur medizinischen Versorgung haben, teurer ist, als in der Gruppe, die de facto einen Zugang zur Regelversorgung hat. Für Asyl- bewerber innerhalb der Zeit, in der nur eingeschränkter Zugang zur medizinischen Versorgung besteht, sind es im Durchschnitt pro Jahr 375,80 Euro mehr. Oder anders bilanziert: Man hätte über den gesamten Untersuchungszeitraum 1,56 Milliarden Euro sparen können, wären da nicht die Einschränkungen nach $\S 4$ und 6 des Asylbewerberleistungsgesetzes.

Wie kommen Sie darauf, dass das mit den Einschränkungen des Asylbewerberleistungsgesetzes zu tun hat? Vielleicht brauchen Flüchtlinge, die neu hier ankommen, in den ersten Monaten einfach mehr medizinische Versorgung und kosten daher mehr?

Das kann sein, ja, aber die Asylbewerberleistungsstatistik enthält keine Daten zur Art der Erkrankung und Behandlung, sodass wir direkt nicht untersuchen können.

Es gibt aber einige Hinweise, die dafür sprechen, dass es eben die Beschränkung der Leistung ist, die eine wichtige Rolle spielen dürfte.

\section{Bitte?}

Diese Wartezeiten haben sich ja wie geschildert im Untersuchungszeitraum von
20 Jahren mehrfach geändert, von 12 Monaten auf 36 und dann 48 Monate. Die Unterschiede in den Kosten zwischen beiden Gruppen ziehen sich aber durch den ganzen Untersuchungszeitraum hindurch, und je länger die Wartezeit, desto größer wurden statistisch die Unterschiede der Kosten. Das ist ein Anhaltspunkt, warum wir sagen, dass eine zentrale Ursache der Mehrkosten eben in dem eingeschränkten Zugang an sich zu liegen scheint. Daneben haben wir nur einen zweiten Faktor gefunden, der ebenfalls einen Teil der Unterschiede erklären könnte.

\section{Als da wäre?}

Es ist die Art der Unterbringung, ob jemand dezentral untergebracht war oder in einer Gemeinschaftsunterkunft lebte. Die Gesundheitsausgaben waren niedriger für die Personen, die dezentral untergebracht waren und so wieder Privatsphäre hatten. Und auch das macht Sinn. Es gibt relativ gute empirische Belege dafür, dass die Unterbringung in Sammelunterkünften Stress bereitet und das Risiko für vermeidbare Infektionserkrankungen erhöht. An erster Stelle aber steht unsere Erkenntnis, dass die Restriktionen der medizinischen Versorgung durch das Asylbewer- 


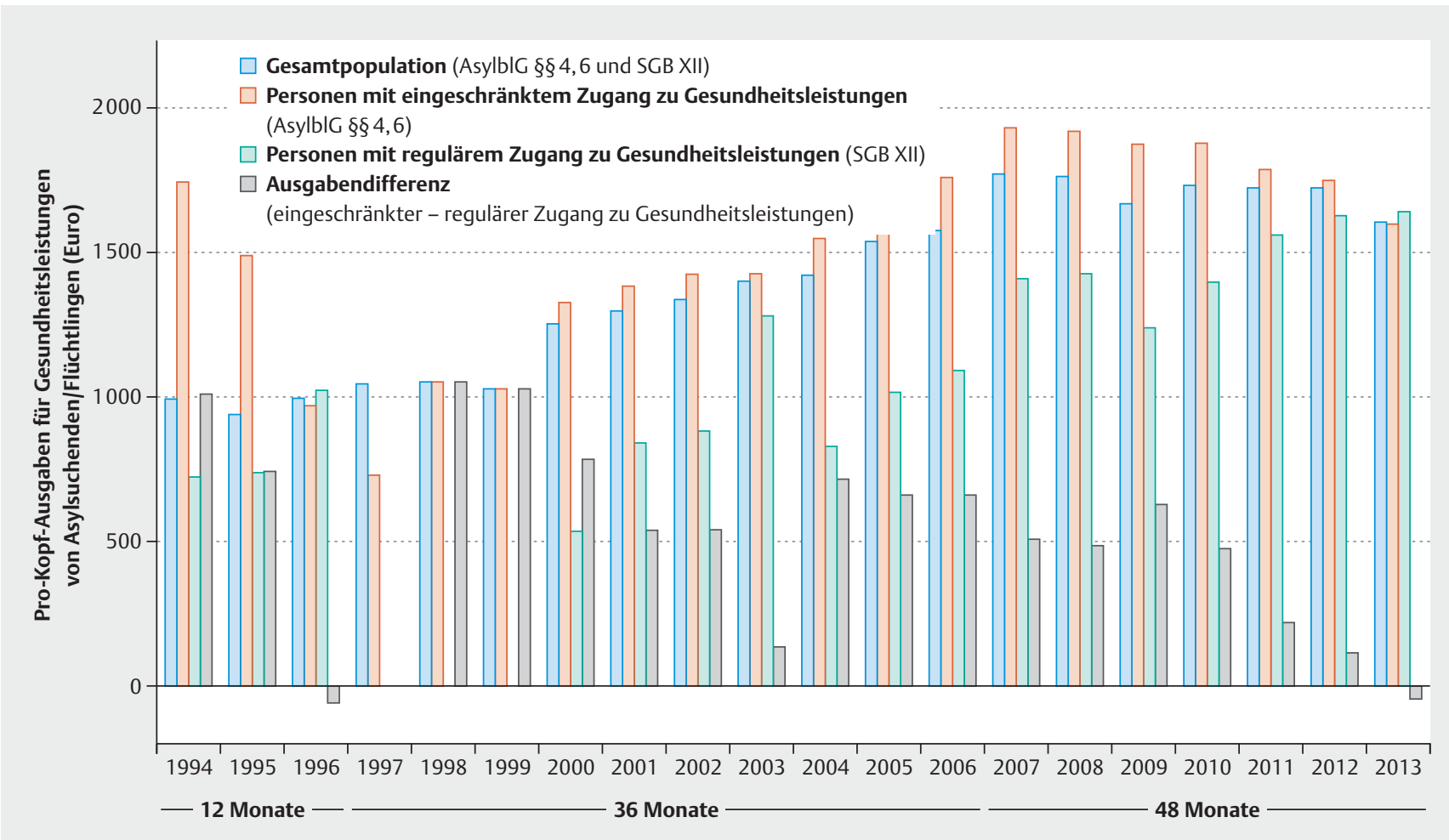

- Abb. 2 Pro-Kopf-Ausgaben für Gesundheitsleistungen.

berleistungsgesetz nicht zu Kosteneinsparungen führen.

Es hat daher meiner Meinung nach nicht nur aus ethischen und medizinischen, sondern auch aus finanziellen Gründen nur Vorteile, Flüchtlingen gleich uneingeschränkten Zugang in die Regelversorgung zu geben. Es wäre richtig, die Einschränkungen im Gesetz abzuschaffen.

\section{Das muss der Bundestag machen.}

Durchaus, das sollte er ändern. Mit dem Asylbewerberleistungsgesetz wird seit 1993 Migrationspolitik im Bereich der Gesundheitspolitik gemacht. Und dies offenkundig mit den falschen Mitteln. Wir haben uns im Gesundheitssystem doch eigentlich längst darauf verständigt, dass Gesundheitspolitik evidenzbasiert planen muss. Es ist aus dieser Sicht generell nicht tragbar, den Zugang zur Medizin einzuschränken. Wir wissen auch aus der $\mathrm{Er}$ fahrung von anderen Ländern, dass ein schlechterer Zugang zur Medizin, dass obendrein Parallelsysteme eigentlich immer teurer und ineffizient sind. Warum soll das hier anders sein?
Generell sagen wir doch heute, dass wir ein Gesundheitssystem brauchen, das Früherkennung fördert, wir brauchen einen guten Zugang zur Versorgung, zur Primärversorgung, koordinative Funktionen, wir müssen kontinuierliche Versorgung leisten, damit wir möglichst viele Krankheiten möglichst frühzeitig behandeln können, was dann eben auch teure stationäre Behandlungen und Folgekosten spart.

Dann stellt sich die Frage, ob das nicht dieselben Maßnahmen sind, die wir auch der Flüchtlingspopulation anbieten müssen. Wenn wir Geld sparen, wenn wir verhindern möchten, dass Menschen zu spät behandelt werden, die früher hätten behandelt werden können.

Manche Stimmen berichten aber, dass die Versorgung in der Praxis trotz der Einschränkungen durch das Asylbewerberleistungsgesetz so schlecht nicht ist (siehe auch das Interview Dehmlow, Z Orthop Unfall Heft 2/2017; 155: 134).

In der Tat sagt das Gesetz am Ende auch, dass diejenigen, die tatsächlich Leistungen brauchen, sie auch bekommen sollen. Und diejenigen, die eine Maßnahme brauchen, bekommen sie auch, das ist schon auch mein Eindruck. Nur dauert es eben viel länger, es ist komplizierter mit viel mehr bürokratischem Aufwand. Und das System tut sich damit keinen Gefallen. Die Kosten für den hohen Aufwand, die erfasst unsere Studie gar nicht. Wie oft hier jemand zwischen Sozialamt, Gesundheitsamt und Arzt hin und her muss, wie oft er dann im Krankenhaus vielleicht auch blockiert, das sind vermutlich weitere Millionen, die bei uns noch gar nicht drin sind und die sich sparen ließen.

Man muss sich auch fragen, warum die Beweislast ausgerechnet bei einer so wichtigen Frage bei denen liegen soll, die eine gesetzliche Einschränkung abschaffen wollen. Ich sehe es genau anders herum. Wenn man eine Maßnahme einführt, sollte man zunächst nachweisen können, dass es funktioniert und dass es sinnvoll ist. Zumal, wenn man Einschränkungen beim Zugang zur medizinischen Versorgung einführt. Dieser Beweis fehlt beim Asylbewerberleistungsgesetz. 
Gibt es weitere Studien, die diesen Zusammenhang beleuchten?

Meines Wissens ist unsere Arbeit bislang die einzige, die versucht, die Kosten des Asylbewerberleistungsgesetzes empirisch zu untersuchen.

Der von Ihnen untersuchte Datensatz endet 2013. Würde sich das Gleiche finden, wenn Sie neuere Daten dazu auswerten? Erst 2015 gab es einen Zustrom besonders vieler Flüchtlinge.

Das wissen wir nicht. Ich finde Projektionen aus solchen Zeitreihen, die auf Routinedaten basieren, schwierig. Es würde Sinn machen, sich die Daten von 2013 bis 2018 anzuschauen, wir haben das später vor.

Was sagen Sie zur Ausgabe einer eGK von Anfang an?

Grundsätzlich soll man alles tun, den Zugang zur Versorgung niederschwellig zu gestalten. Und der Umweg über das Sozialamt kann durch die eGK eingespart werden. Allein aus dieser Perspektive sage ich, ist die eGK eine Win-win-Situation.
Allerdings hat sich die Szene leider hier ein Zusatzproblem eingefangen, das hausgemacht ist. Viele Kommunen führen die Karte aktuell nicht ein, weil die Kassen für die Verwaltung einen vergleichsweise hohen Zusatzbonus verlangen. Da hat man es verpasst, bundesweit dafür eine Lösung zu finden.

Was ist eigentlich mit den Patientenakten bei Flüchtlingen?

Das ist ein großes Problem. Viele Gesundheitsinformationen gehen unter, kommen nicht an, bleiben zwischen den Sektoren hängen.

Angenommen, ein Patient mit Lungenproblemen kommt ein halbes Jahr nach Aufenthaltsbeginn zu einem niedergelassenen Arzt. Gibt es eine Chance, eine Röntgenaufnahme, die womöglich bei der Erstuntersuchung gemacht wurde, zu bekommen?

Wenn Sie eruieren können, wo jemand nun geröntgt wurde, dann vielleicht. Mit einer der Asylgesetznovellen ist jetzt vorgesehen, dass die Ergebnisse aus Unter- suchungen alle im Ausländerzentralregister dokumentiert werden sollen. Zugriff haben bisher aber nur Behörden.

Wir haben uns deshalb vor einiger Zeit eine schnellere Lösung als Soforthilfe überlegt. Es ist ein kleines Patientenheft, das dem Patienten mitgegeben wird, in dem die wesentlichen Informationen notiert werden, was wo gemacht wird. Im Regierungsbezirk Karlsruhe erhalten es bereits alle Asylbewerber. Im Laufe des Jahres soll das auch in den anderen Regierungsbezirken von Baden-Württemberg eingeführt werden. Das erlaubt zumindest festzustellen, wo man nach womöglich vorhandenen Unterlagen fahnden kann.

\section{Literatur}

[1] Bozorgmehr K, Razum O. Effect of Restricting Access to Health Care on Health Expenditures among Asylum-Seekers and Refugees: A Quasi-Experimental Study in Germany, 1994-2013. PLoS One 2015; DOI: 10.1371/ journal.pone.0131483

Das Interview führte Bernhard Epping. 